\title{
Aspects on radiolabeling of 177Lu-DOTA-TATE: After C18 purification re-addition of ascorbic acid is required to maintain radiochemical purity
}

\author{
Stephan Maus ${ }^{1}$, Erik de Blois ${ }^{2}$, Stephan J. Ament ${ }^{1}$, Mathias Schreckenberger ${ }^{1}$, Wouter A. P. \\ Breeman $^{2}$
}

1. Clinic of Nuclear Medicine, University Medical Centre Mainz, Langenbeckstr, Mainz, Germany. 2. Department of Nuclear Medicine, Erasmus MC Rotterdam, Rotterdam, Netherlands

Correspondence: Stephan Maus. Address: Klinik und Poliklinik für NuklearmedizinUniversitätsmedizin der Johannes Gutenberg-Universität Mainz, Germany. Email: stephan.maus@unimedizin-mainz.de

Received: January 24, 2014

Accepted: February 16, 2014

DOI : $10.5430 /$ ijdi.v1n1p5

URL: http://dx.doi.org/10.5430/ijdi.v1n1p5

Online Published: February 21, 2014

\section{Abstract}

Purpose: Radiolabeled peptides like 177Lu-DOTA-TATE are vulnerable to radiolysis, which results in decreased radiochemical purity (RCP) of these radiopeptides. Gentisic acid (GA) and ascorbic acid (AA) are well known ingredients to reduce the effects of radiolysis. Currently, there is a trend to change the procedure from a manual to a cassette-based automated labeling and to introduce a C18 solid phase extraction (SPE) post-radiolabeling in order to remove non-incorporated $177 \mathrm{Lu}$ from the injection solution. However, with the introduction of SPE purification, GA and AA might effectively be removed from injection solution with a concordant dramatically drop of the RCP. Therefore we investigated the impact of tC18 SPE purification on the RCP of 177Lu-DOTA-TATE.

Methods: We compared the manual radiolabeling procedure with the cassette-based automated radiolabeling procedure with/out tC18 SPE purification cartridge. The effect of tC18 purification on RCP of 177Lu-DOTA-TATE was investigated by HPLC as function of the post-radiolabeling time and the concentration of activity.

Results: After tC18 SPE purification, GA and AA were effectively removed and resulted in volume-dependent decrease in RCP, e.g. $<95 \%$ after $5 \mathrm{~h}$ in $20 \mathrm{~mL}$. Re-addition of AA directly after tC18 SPE purification resulted in a RCP $\geq 95 \%$ at $72 \mathrm{~h}$. In addition, with the cassette-based automated radiolabeling procedure we also found $28 \%$ of the original activity remaining in the activity-containing vial and tubing vs. $<1 \%$ with the manual procedure.

Conclusion: Re-addition of AA post tC18 SPE purification is required to maintain RCP of 177Lu-DOTA-TATE.

\section{Keywords}

Lutetium-177, 177Lu-DOTA-TATE, Cassette-based, Automated radiolabeling, Radiochemical purity, Ascorbic acid, Quencher, Gentisic acid, Radiolysis

\section{I ntroduction}

Radiolabeled somatostatin analogues, such as [DOTA0,Tyr3]octreotate, further referred as DOTA-TATE have been subject of intensive research during the last 2 decades and play an important role in somatostatin receptor imaging and peptide receptor-targeted radionuclide therapy (PRRT) e.g. 177Lu-DOTA-TATE ${ }^{[1-7]}$. 
RCP of 177Lu-DOTA-TATE is an essential factor for successful PRRT. Because of the high doses of 177Lu-DOTA-TATE (7.4 GBq 177Lu per PRRT administration), the peptide is subject to radiolysis. The degree of radiolysis is influenced by several factors like the amount of DOTA-TATE, temperature, time, the total activity, the volumic activity, quencher(s) et cetera ${ }^{[8-12]}$.

In the current publication we present a comparative study to investigate the effect of gentisic acid (GA) and ascorbic acid (AA) as quenchers during and after (manual and cassette-based automated) the radiolabeling 177Lu-DOTA-TATE procedures.

There is currently a trend to move from manual radiolabeling to a cassette-based automated radiolabeling procedure; therefore we investigated these two different procedures in parallel. Sep-Pak Light tC18 SPE purification (further referred as $\mathrm{tC} 18$ ) is included as default in the cassette-based automated procedure. However, the $\mathrm{tC} 18$ purification of the reaction mixture after radiolabeling potentially removes GA and/or AA effectively. The aim of this study was to compare radiolabeling procedures (manual vs. cassette-based automated) with and without $\mathrm{tC} 18$ purification and to investigate the impact on the RCP of 177Lu-DOTA-TATE as function of time until the moment of administration to patient.

\section{Materials and chemicals}

Reagents and solvents were used in the highest quality grade without further purification.

GA and water for trace analyses (Trace-SELECT ${ }^{\circledR}$ ) were purchased from Sigma-Aldrich Chemie GmbH (Taufkirchen, Germany). Hydrochloric acid 30\% ( $\mathrm{HCl})$ Ultrapur was obtained from Merck KGaA (Darmstadt, Germany). AA was purchased from WÖRWAG Pharma GmbH \& Co. KG (Böblingen, Germany). DOTA-TATE as kit formulation [13] was provided by Erasmus MC Rotterdam (Rotterdam, The Netherlands). Ethanol (99.5\%), Aqua ad iniectabilia and isotonic $0.9 \% \mathrm{NaCl}$ (further referred as saline) were purchased from B.Braun Melsungen AG (Melsungen, Germany). tC18 cartridges were obtained from Waters $\mathrm{GmbH}$ (Eschborn, Germany). 177 $\mathrm{LuCl} 3$ with specific activities in the range 740-1000 GBq/mg was bought from IDB-Holland (Baarle Nassau, the Netherlands).

\section{General methods}

The manual radiolabeling procedure was performed in a temperature-controlled heating block from CardiRad (Lohja, Finland) and cassette-based automated radiolabeling procedure was performed in a Modular-Lab Pharm Tracer module (EZAG, Berlin, Germany) using the C4-Y90-00-standard synthesis and C1-PR-00 pressure test cassette (EZAG, Berlin, Germany).

\subsection{Cassette-based automated radiolabeling procedure}

The cassette was prepared according to the Y-Lu-INCASSETTE-TEST protocol (EZAG, Berlin, Germany). For standard patient radiolabeling, $240 \mu \mathrm{g}$ DOTA-TATE in $0.6 \mathrm{~mL}(400 \mu \mathrm{g} / \mathrm{mL})$ of the DOTA-TATE kit formulation ${ }^{[13]}$ was transferred automatically and quantitatively in to a glass reaction vial (RV) and eventually $7.5 \mathrm{GBq} 177 \mathrm{LuCl} 3(0.3 \mathrm{~mL})$ was added in to RV. Subsequent program steps are consecutively executed and the radiolabeling was running automatically according to the LU-177-DOTA-PEPTIDES-PT-V.X.X protocol (according EZAG). RV was heated for 30 min at $80^{\circ} \mathrm{C}$. After 5 min cooling to ambient temperature the reaction mixture was transferred from the RV to the preconditioned tC18 cartridge. Pre-conditioning of the tC18 cartridge was performed with an ethanol/water mixture (5 $\mathrm{mL}, 50: 50 \% \mathrm{v} / \mathrm{v}$ ). This $\mathrm{tC} 18$ procedure was introduced in order to remove non-incorporated $177 \mathrm{Lu}$ from the final product. The content of RV was transferred to the $\mathrm{tC} 18$ cartridge and rinsed with $3 \mathrm{~mL}$ saline. 177Lu-DOTA-TATE was desorbed from the tC18 cartridge with $2.5 \mathrm{~mL}$ of ethanol/water $(50: 50 \% \mathrm{v} / \mathrm{v})$. An aliquot of the final mixture was taken and subjected to quality control by ITLC and HPLC. The eluate plus $17.5 \mathrm{~mL}$ saline solution (final volume $20 \mathrm{~mL}$ ) were 
filtered with means of a sterile Millex-GV $0.22 \mu \mathrm{m}$ filter into a $25 \mathrm{~mL}$ glass vial (product vial or PV). Finally, a filter integrity test was performed. The cassette-based automated radiolabeling procedure process takes about 60 min.

\subsection{Manual radiolabeling procedure}

The manual radiolabeling procedure was performed directly in the activity-containing vial (AV), containing $7.5 \mathrm{GBq}$ $177 \mathrm{LuCl} 3$ in $0.3 \mathrm{~mL}$. For standart patient radiolabeling $0.6 \mathrm{~mL}$ ( $240 \mu \mathrm{g}$ DOTA-Tate) of the DOTA-TATE kit formulation ${ }^{[13]}$ was added to the $\mathrm{AV}$, and incubated for $30 \mathrm{~min}$ at $80{ }^{\circ} \mathrm{C}{ }^{[13]}$. After cooling down to ambient temperature non-incorporated $177 \mathrm{Lu}$ was complexed by the addition of $0.25 \mathrm{~mL}$ DTPA-solution $(4 \mathrm{mg} / \mathrm{mL})$ to the reaction mixture ${ }^{[14-15]}$. An aliquot of the reaction mixture was taken and quality control was performed using ITLC and HPLC. The residual was diluted with $5 \mathrm{~mL}$ saline solution, filtered with means of a sterile Millex-GV $0.22 \mu \mathrm{m}$ filter into PV and finally adjusted to a final volume of $20 \mathrm{~mL}$. The manual radiolabeling procedure takes about $40 \mathrm{~min}$.

\section{Studies on RCP of 177 Lu-DOTA-TATE}

RCP is defined as the \% of the activity of the radionuclide present in the desired radiopharmaceutical form of the total radioactivity. RCP of 177Lu-DOTA-TATE was investigated with/out tC18 purification and re-addition of quenchers as shown in experiments 1-4, see Table 1. In order to investigate the dilution of the quenchers two additional radiolabeling were performed (experiment 5-6, see Table 1). One radiolabeling included tC18 purification (experiment 5, see Table 1), while another radiolabeling (experiment 6) was performed without the tC18 purification. Both samples were diluted with saline up to a final volume of $20 \mathrm{~mL}$, at constant concentration of $\sim 0.5 \mathrm{GBq} / \mathrm{mL}$. In addition, experiment 7 was performed without $\mathrm{tC} 18$ purification post radiolabeling and was diluted to a patient dose (7.4 GBq in $100 \mathrm{~mL})$. RCP was determined by HPLC, as described below.

Table 1. Different post radiolabeling procedures of 177Lu-DOTATATE

\begin{tabular}{llllllll}
\hline Experiment & $\mathbf{1}$ & $\mathbf{2}$ & $\mathbf{3}$ & $\mathbf{4}$ & $\mathbf{5}$ & $\mathbf{6}$ & $\mathbf{7}$ \\
\hline $\mathrm{tC}_{18}$ purification & - & + & + & + & - & + & - \\
Ethanol/ $\mathrm{H}_{2} \mathrm{O} 50 / 50 \%(2.5 \mathrm{~mL})$ & - & + & + & + & - & & + \\
Re-addition AA or GA & - & - & $\mathrm{AA}$ & $\mathrm{GA}$ & - & - & - \\
$(100 \mathrm{mmol} / \mathrm{L})$ & 5 & 5 & 5 & 5 & 20 & 20 & 100 \\
Final volume $(\mathrm{mL})$ & 2.5 & 2.5 & 2.5 & 2.5 & 10 & 10 & 7.4 \\
Total activity $(\mathrm{GBq})$ & + & + & + & + & + & + & - \\
$0.5 \mathrm{GBq} / \mathrm{mL}$ & & & & & & \\
\hline
\end{tabular}

Labelings were performed using $7.5 \mathrm{GBq}$ of $177 \mathrm{LuCl} 3(0.3 \mathrm{~mL})$ and $0.6 \mathrm{~mL}$ of kit Erasmus MC matrix. Thereafter post radiolabeling procedures were performed either with/out tC18 purification, re-addition of AA or GA, and diluted in different final volumes $(5,20$ and $100 \mathrm{~mL})$ with saline. RCP of 177Lu-DOTA-TATE was monitored as function of time

\section{Analytical methods}

\subsection{I ncorporation by I TLC}

ITLC-SG glass fibre sheets were purchased from PALL Life Sciences (Port Washington, NY, USA). Small portions (1-3 $\mu \mathrm{L})$ were added on the ITLC-SG strips and sodium citrate buffer $(0.1 \mathrm{M}, \mathrm{pH} 5)$ was used as mobile phase as described ${ }^{[14,16]}$. Activity was recorded by Gina Star TLC and analyzed using Raytest miniGita software (Straubenhardt, Germany). Calculation of incorporation was performed as described ${ }^{[16]}$. 


\subsection{HPLC}

HPLC methods were performed using a Dionex-3000 HPLC system with a variable wavelength Dionex GmbH detector (Idstein, Germany) containing a Waters $4.6 \mathrm{~mm} \times 250 \mathrm{~mm}, 5 \mu \mathrm{m}$ Symmetry C18 column (Eschborn, Germany). The gradient elution system utilized mobile phase $\mathrm{A}$ (methanol) and mobile phase $\mathrm{B}(0.06 \mathrm{M}$ sodium acetate buffer, $\mathrm{pH}$ 5.5). Gradient was performed with a flow rate of $1 \mathrm{~mL} / \mathrm{min}$ starting with $100 \% \mathrm{~B}$ for $6.5 \mathrm{~min}$; and was changed to $50 \% \mathrm{~A}$ and $50 \% \mathrm{~B}$ within $0.5 \mathrm{~min}$ and increased to $60 \% \mathrm{~A}$ over $20 \mathrm{~min}$.

Thereafter, the mobile phase A was increased within 0.2 min to $100 \%$ and was kept constant for 4.8 min. Finally, the gradient parameters returned to the initial starting conditions. The data were analyzed using Chromeleon Client Software Version 6.8.9. from Dionex GmbH (Idstein, Germany).

\section{Results}

\subsection{Radiochemical yield}

With the cassette-based automated radiolabeling procedure we obtained radiochemical yields of $71 \pm 18 \%(\mathrm{n}=12)$. The manual labeling achieved radiochemical yields $\geq 99 \%$ ( $n=3$ ), independent of activity (range 2.5-10 GBq) or final volume (range $5-20 \mathrm{~mL}$ ).

\subsection{Radiolabeling without tC18 Purification}

RCP of 177Lu-DOTA-TATE was measured by HPLC up to $168 \mathrm{~h}$ post-radiolabeling. Fig. 1A shows a typical HPLC radiochromatogram of 177Lu-DOTA-TATE which was prepared as described ${ }^{[13]}$. Fig. 1B shows the HPLC radiochromatogram of 177Lu-DOTA-TATE in the absence of quenchers. Fig. 2A clearly demonstrates that 177Lu-DOTA-TATE without the tC18 purification post-radiolabeling remained stable (RCP $\geq 95 \%$ at $72 \mathrm{~h}$ post radiolabeling, experiment 1 and 2, see Table 1).
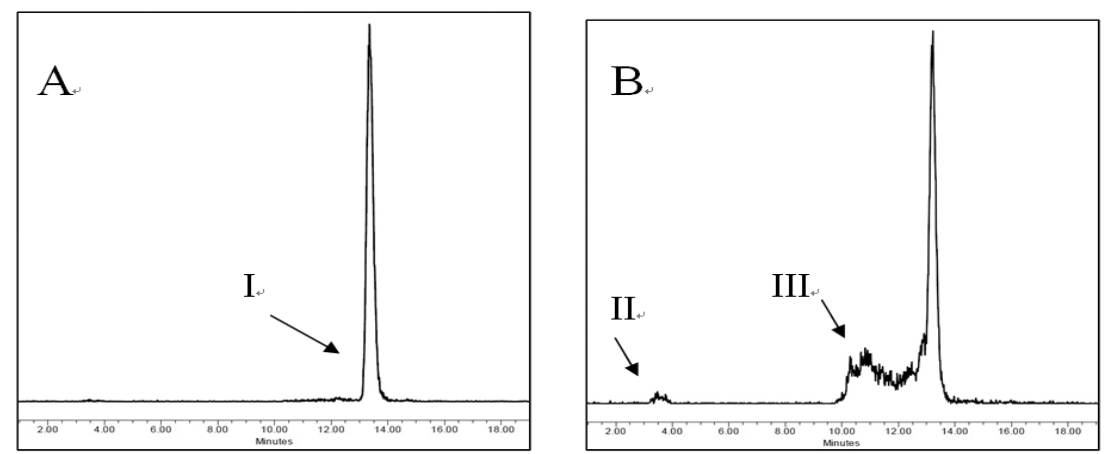

Figure 1. Typical RP-HPLC radiochromatogram of 177Lu-DOTA-TATE

(A) with a RCP of $>95 \%$ and ${ }^{177}$ Lu-DOTA-TATE (B) with a RCP $<95 \%$. Peaks (I): ${ }^{177}$ Lu-DOTA-TATE, (II): ${ }^{177}$ Lu-DTPA and ${ }^{177} \mathrm{Lu}$ and (III): radiolysed fragments of ${ }^{177} \mathrm{Lu}$-DOTA-TATE. These fragments were not further characterised. X-axes are expressed in time ( $\mathrm{min})$ and $\mathrm{Y}$-axes in $\mathrm{mV}$.

After increasing the volume up to $20 \mathrm{~mL}$ at constant volume activity (experiments 5, Table 1), 177Lu-DOTA-TATE remained stable ( $\mathrm{RCP} \geq 95 \% \sim 24 \mathrm{~h}$ post-radiolabeling) as shown in Fig. 2B, while the RCP of 177Lu-DOTA-TATE in a patient dose $(7.4 \mathrm{GBq} / 100 \mathrm{~mL}$ ) rapidly decreased below $95 \%$ within $12 \mathrm{~h}$ (Fig 2C, experiment 7, see Table 1). 


\subsection{Radiolabeling with $\mathbf{t C}_{18}$ purification}

tC18 purification of reaction mixture containing 177Lu-DOTA-TATE totally removed both AA and GA (>99\%), as confirmed by HPLC (data not shown). The tC18 purified fraction without re-addition of quencher in total volume of $5 \mathrm{~mL}$ resulted in a $95 \% \mathrm{RCP}$ after $\sim 35 \mathrm{~h}$ and $92 \%$ at $72 \mathrm{~h}$ post radiolabeling (Fig. 2A, experiment 2, see Table 1). While RCP of 177Lu-DOTA-TATE in $20 \mathrm{~mL}$ final volume decreased much more rapidly and resulted in a RCP of $<95 \%$ after $\sim 5 \mathrm{~h}$ and $74 \%$ at $24 \mathrm{~h}$ post radiolabeling (Fig. 2B, experiment 6, see Table 1).
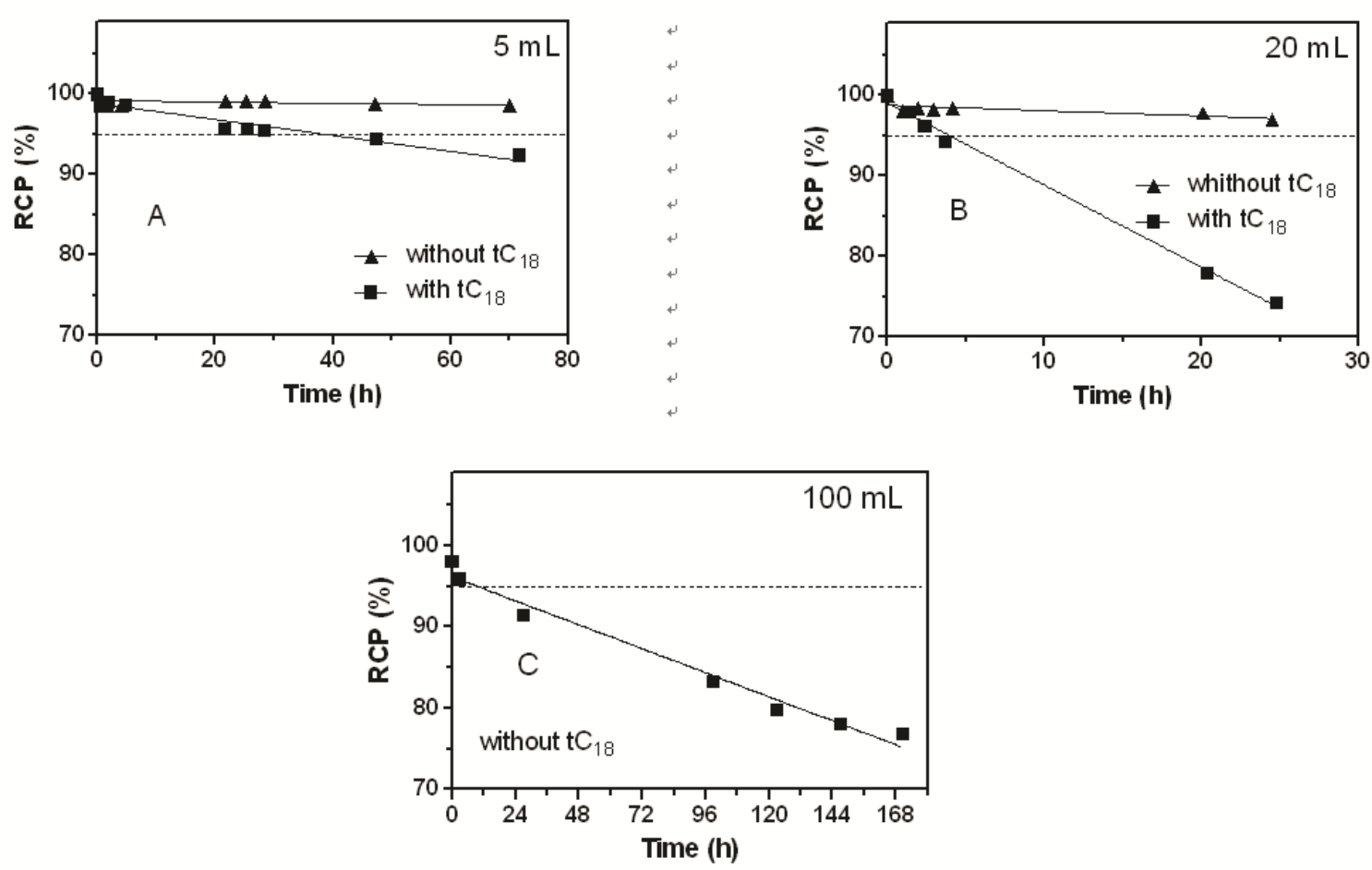

Figure 2. RCP of tC18 purified 177Lu-DOTA-TATE as function of time in a final volume

$5 \mathrm{~mL}$ (A, see experiments 1 and 2, Table 1) and $20 \mathrm{~mL}$ (B, see experiments 5 and 6, Table 1), both at $0.5 \mathrm{GBq} / \mathrm{mL}$. (C): represents a patient dose $(7.4 \mathrm{GBq} / 100 \mathrm{~mL})$ without $\mathrm{tC}_{18}$ purification diluted with saline to a final volume of $100 \mathrm{~mL}$ (experiment 7, Table 1). X-axes are expressed in time (h) and Y-axes in RCP (\%). Dotted lines represents 95\% RCP and is taken as lowest level suitable for patient administration.

\subsection{Re-addition of AA or GA post $\mathrm{tC}_{18}$ purification}

$\mathrm{RCP}$ of $177 \mathrm{Lu}-\mathrm{DOTA}$-TATE with the re-addition of AA ( $100 \mathrm{mmol} / \mathrm{L})$ post $\mathrm{tC} 18$ purification was $\geq 95 \%$ at $\sim 72 \mathrm{~h}$ post radiolabeling (Fig. 3, experiment 3, see Table 1). Re-addition of GA (100 mmol/L, experiment 4, see Table 1) had only minor stabilizing properties, RCP of 177Lu-DOTA-TATE decreased below $<95 \%$ within $\sim 24 \mathrm{~h}$ post radiolabeling (data not shown). Fragments observed in HPLC radiochromatogram as peaks prior to the main peak were caused by radiolysis of 177Lu-DOTA-TATE (Fig. 1B), were not further characterised. Fragments observed were not caused by formation of ionic 177Lu, 177Lu-DTPA or 177Lu-DOTA, since they have no retention on C18 column and are eluted from the HPLC system directly after void volume (Fig 1B, fragment II).

\subsection{Activity loss, localisation of activity}

Performing the manual radiolabeling including $\mathrm{tC}_{18}$ purification resulted in $5.2 \pm 0.5 \%(\mathrm{n}=3)$ loss of activity in the $\mathrm{tC}_{18}$ purification cartridge. Whereas with the cassette-based automated radiolabeling procedure, loss of activity in the cassette was $28 \pm 18 \%(\mathrm{n}=12)$, mainly caused by adhesion in the $\mathrm{RV}$, tubing and $\mathrm{tC}_{18}$ cartridge. 


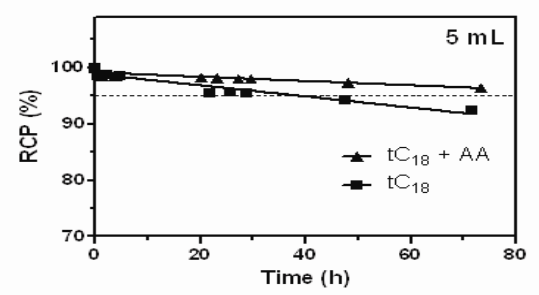

Figure 3. $\mathrm{RCP}$ of $\mathrm{tC}_{18}$ purified ${ }^{177} \mathrm{Lu}$-DOTA-TATE as a function of time in a total volume $5 \mathrm{~mL}$ with/out re-addition of AA (see experiments 2 and 3, Table 1). X-axes are expressed in time (h) and Y-axes in RCP (\%). The dotted line represents $95 \% \mathrm{RCP}$ and is taken as lowest level suitable for patient administration.

\section{Discussion}

Liu et al. reported the addition of GA and AA as quenchers for radiolabeled DOTA-biomolecules in order to prevent radiolysis ${ }^{[8,10]}$. They clearly demonstrated factors which influence $\mathrm{RCP}$, factors such as presence and the relative amount of quencher, as well as the activity amount and the activity/quencher ratio. These data are in accordance with our studies. AA and GA-containing DOTA-TATE kit formulation was used and radiolabeled as described ${ }^{[13]}$. With regards to the production of radiopharmaceuticals, the existing GMP requirements as well as the reduction of the radiation exposure for the employees, Petrik et al. ${ }^{[18]}$ reported radiolabeling of peptides for diagnostic and therapeutic purposes using a cassette-based automated radiolabeling procedure. They also showed radiochemical yields of $89 \%$ and $\mathrm{RCP}$ of $\geq 95 \%$, however, without presenting data on RCP as function of time post radiolabeling, whereas in daily practice radiolabeled peptides will be administered hours after radiolabeling. We used the same cassette-based automated radiolabeling procedure and a DOTA-TATE kit formulation and were also able to maintain RCP at $\geq 95 \%$. However, we observed $28 \pm$ $18 \%$ loss of activity in the cassette-based procedure $(\mathrm{n}=12)$, and, in addition, a decrease of $\mathrm{RCP}$ after $\mathrm{tC} 18$ purification as function of the volumic activity.

To investigate this phenomenon, we performed parallel manual and cassette-based automated radiolabeling syntheses. In the latter, $\mathrm{tC} 18$ purification is included as a safty-net system, to remove possible to free non-incorporated $177 \mathrm{Lu}$ ions from the reaction solution. Therefore, a tC18 purification step was also included in the manual radiolabeling. In two identical performed radiolabeling syntheses the initial RCP was $\geq 95 \%$, however, since tC18 purification of eluate after radiolabeling resulted in total elimination of AA and GA and resulted in a decreased RCP of $<95 \%$ (Fig. 2A, experiments 1 and 2, Table 1). The experiments were repeated and after tC18 purification step GA or AA (final concentrations of 100 $\mathrm{mmol} / \mathrm{L}$ ) were added directly in to $177 \mathrm{Lu}-\mathrm{DOTA}-\mathrm{TATE}$ containing labeling mixture (experiments 3 and 4 , Table 1).

Additional experiments were performed in $20 \mathrm{~mL}$ total volume, (experiments 5 and 6 , see Table 1), while keeping the volume activity constant $(0.5 \mathrm{GBq} / \mathrm{mL})$. These experiments showed a much more rapidly decrease in RCP of 177Lu-DOTA-TATE ( $<95 \%$ RCP after $\sim 5 \mathrm{~h}$ and $74 \%$ at $\sim 24 \mathrm{~h}$ post radiolabeling, Fig. $2 \mathrm{~B}$,). Further dilution $(5 \rightarrow 20 \mathrm{~mL})$ resulted in concordant dilution of quencher and might explain the decrease of RCP of 177Lu-DOTA-TATE.

As shown in Fig. 2A and B, experiments 2 and 6, Table 1, both after $\mathrm{C} 18$ purification, we found a more rapid decrease in $\mathrm{RCP}$ in $20 \mathrm{~mL}$ vs $5 \mathrm{~mL}$. Without $\mathrm{C} 18$ purification the difference in decrease of RCP was less clear, experiments 1 and 5 , Table 1, Fig. 2A and B. However, there is a difference between experiments 2 and 6: after the C18 purification (and $2.5 \mathrm{~mL}$ (50:50 water:ethanol)) the residual is diluted with $2.5 \mathrm{~mL}$ saline solution (experiment 2) or $17.5 \mathrm{~mL}$ saline solution (experiment 6). In consequence, the final ethanol concentration is different, $0.25 \mathrm{~mL}$ ethanol per $\mathrm{mL}$ or $0.062 \mathrm{~mL}$ ethanol per $\mathrm{mL}(1 \mathrm{mmoles} / \mathrm{L}$ ethanol in experiment $2(5 \mathrm{~mL})$ and $0.25 \mathrm{mmoles} / \mathrm{L})$ in experiment $6(20 \mathrm{~mL})$. 
Based on these results we can conclude that the standart implementation of a tC18 purification, which are generally effective for eliminating non-incorporated $177 \mathrm{Lu}$ ions, needs the re-addition of a quencher to maintain RCP of 177Lu-DOTA-TATE, although when ethanol is also known as an effective quencher in the mmoles/L range ${ }^{[17-18]}$.

In daily practice, performing a manual radiolabeling as described ${ }^{[13]}$, without $\mathrm{tC} 18$ purification, patient doses are diluted to $100 \mathrm{~mL}$ with saline. However, without the re-addition of quenchers, the RCP decreases time-dependent, while the RCP could be maintained $>95 \%$ up to $12 \mathrm{~h}$ after radiolabeling (Fig. 2C). Thus, the dilution to $100 \mathrm{~mL}$ after radiolabeling, even without using tC18 purification, is preferably performed by the addition of AA up to a final concentration of $100 \mathrm{mmol} / \mathrm{L}$. The re-addition of GA under the same conditions showed only minor stabilizing properties. To our knowledge RCP data on GA addition on $177 \mathrm{Lu}$-labeled DOTA-peptides are not available in literature, however, Liu et al. ${ }^{[8,10]}$ reported a beneficial effect on the addition of GA to 90Y-DOTA-biomolecules.

When therapeutic doses of 177Lu-DOTA-TATE are administered (7.4 GBq for PRRT), radiolysed 177Lu-labeled fragments will most likely not bind to somatostatin receptor-positive tumor tissue. Here, however, it should be clarified, that $1 \%$ loss in RCP represents $74 \mathrm{MBq} 177 \mathrm{Lu}$ non-characterised radiolysed peptide (and $370 \mathrm{MBq}$ at $95 \% \mathrm{RCP}$ ), adding undesired radiation dose burden to the patient. As a consequence, we strongly advise re-addition of AA post tC18 purification. In addition, dilution after radiolabeling, even without using tC18 purification, should be performed with addition of AA.

Although not investigated here, the decrease in RCP of all 177Lu-labeled DOTA-biomolecules in the absence of quencher can be anticipated, and thus requires further study ${ }^{[17]}$.

\section{Conclusion}

Re-addition of AA post tC18 SPE purification is required to maintain RCP of 177Lu-DOTA-TATE. RCP of tC18 purified 177Lu-DOTA-TATE, either labeled manually or cassette-based automated, decreases time- and volume-dependent.

\section{Conflict of interests}

The authors declare no conflicts of interests.

\section{References}

[1] Bakker W H, Breeman W A, Kwekkeboom D J, De Jong L C, Krenning E P. Practical aspects of peptide receptor radionuclide therapy with [177Lu][DOTA0, Tyr3] octreotate. Q J Nucl Med Mol Imaging. 2006; 50: 265-71.

[2] De Araujo E B, Caldeira Filho J S, Nagamati L T, Muramoto E, Colturato M T, Couto R M, Pujatti P B, Mengatti J, Silva C P. A comparative study of $131 \mathrm{I}$ and $177 \mathrm{Lu}$ labeled somatostatin analogues for therapy of neuroendocrine tumours. Appl Radiat Isot. 2009; 67: 227-33. http://dx.doi.org/10.1016/j.apradiso.2008.09.009

[3] De Jong M, Breeman W A, Valkema R, Bernard B F, Krenning E P. Combination radionuclide therapy using 177Lu- and 90Y-labeled somatostatin analogs. J Nucl Med. 2005; 46 Suppl 1: 13S-7S.

[4] De Jong M, Valkema R, Jamar F, Kvols L K, Kwekkeboom D J, Breeman W A, Bakker W H, Smith C, Pauwels S, Krenning E P. Somatostatin receptor-targeted radionuclide therapy of tumors: preclinical and clinical findings. Semin Nucl Med .2002; 32: 133-40. http://dx.doi.org/10.1053/snuc.2002.31027

[5] Kwekkeboom D J, Kam B L, van Essen M, Teunissen J J, van Eijck C H, Valkema R, de Jong M, de Herder W W, Krenning E P. Somatostatin-receptor-based imaging and therapy of gastroenteropancreatic neuroendocrine tumors. Endocr Relat Cancer. 2010; 17: R53-73. http://dx.doi.org/10.1677/ERC-09-0078

[6] Rodriguez-Cortes J, De Murphy C A, Ferro-Flores G, Pedraza-Lopez M, Murphy-Stack E. Biokinetics and dosimetry with Lu-177-DOTA-TATE in athymic mice with induced pancreatic malignant tumours: preclinical studies. Radiation Effects and Defects in Solids. 2007; 162: 791-796. http://dx.doi.org/10.1080/10420150701482568 
[7] Sainz-Esteban A, Prasad V, Schuchardt C, Zachert C, Carril J M, Baum R P. Comparison of sequential planar 177Lu-DOTA-TATE dosimetry scans with 68Ga-DOTA-TATE PET/CT images in patients with metastasized neuroendocrine tumours undergoing peptide receptor radionuclide therapy. Eur J Nucl Med Mol Imaging. 2012; 39: 501-11. http://dx.doi.org/10.1007/s00259-011-2003-x

[8] Liu S, Edwards D S. Stabilization of (90)y-labeled DOTA-biomolecule conjugates using gentisic acid and ascorbic acid. Bioconjug Chem. 2001; 12: 554-8. http://dx.doi.org/10.1021/bc000145v

[9] Liu S, Edwards D S. Bifunctional chelators for therapeutic lanthanide radiopharmaceuticals. Bioconjug Chem. 2001 ; 12 : 7-34. http://dx.doi.org/10.1021/bc000070v

[10] Liu S, Ellars C E, Edwards D S. Ascorbic acid: useful as a buffer agent and radiolytic stabilizer for metalloradiopharmaceuticals. Bioconjug Chem. 2003; 14: 1052-6. http://dx.doi.org/10.1021/bc034109i

[11] Schroeder R P, Muller C, Reneman S, Melis M L, Breeman W A, de Blois E, Bangma C H, Krenning E P, van Weerden W M, de Jong M. A standardised study to compare prostate cancer targeting efficacy of five radiolabelled bombesin analogues. Eur J Nucl Med Mol Imaging. 2010; 37: 1386-96. http://dx.doi.org/10.1007/s00259-010-1388-2

[12] Vaidyanathan G, Affleck D J, Zhao X G, Keir S T, Zalutsky M R. [Lu]-DOTA-Tyr-octreotate: A Potential Targeted Radiotherapeutic for the Treatment of Medulloblastoma. Curr Radiopharm. 2010; 3: 29-36. http://dx.doi.org/10.2174/1874471011003010029

[13] Kwekkeboom D J, Bakker W H, Kooij P P, Konijnenberg M W, Srinivasan A, Erion J L, Schmidt M A, Bugaj J L, de Jong M, Krenning E P. [177Lu-DOTAOTyr3]octreotate: comparison with [111In-DTPAo]octreotide in patients. Eur J Nucl Med. 2001; 28: 1319-25. http://dx.doi.org/10.1007/s002590100574

[14] Breeman W A, van der Wansem K, Bernard B F, van Gameren A, Erion J L, Visser T J, Krenning E P, de Jong M. The addition of DTPA to [177Lu-DOTA0,Tyr3] octreotate prior to administration reduces rat skeleton uptake of radioactivity. Eur J Nucl Med Mol Imaging. 2003; 30: 312-5. http://dx.doi.org/10.1007/s00259-002-1054-4

[15] Seregni E, Maccauro M, Coliva A, Castellani M R, Bajetta E, Aliberti G, Vellani C, Chiesa C, Martinetti A, Bogni A, Bombardieri E. Treatment with tandem [(90)Y]DOTA-TATE and [(177)Lu] DOTA-TATE of neuroendocrine tumors refractory to conventional therapy: preliminary results. Q J Nucl Med Mol Imaging. 2010; 54: 84-91.

[16] Breeman W A P, de Blois E, Bakker W H, Krenning E P, Radiolabeling DOTA-peptides with 90Y and 177Lu to a high specific activity, in Radionuclide Peptide Cancer Therapy, Chinol M,Paganelli G, Editors. 2006, Taylor \& Francis. p. 119-125. http://dx.doi.org/10.3109/9781420016727-6

[17] De Blois E, Chan H S, Konijnenberg M, de Zanger R, Breeman W A. Effectiveness of Quenchers to Reduce Radiolysis of 111In or 177Lu Labelled Methionin-Containing Regulatory Peptides. Maintaining Radiochemical Purity as Measured by HPLC. Curr Top Med Chem. 2013. http://dx.doi.org/10.2174/1568026611212230005

[18] Fukumura T, Nakao R, Yamaguchi M, Suzuki K. Stability of C-11-labeled PET radiopharmaceuticals. Applied Radiation and Isotopes. 2004; 61: 1279-1287. http://dx.doi.org/10.1016/j.apradiso.2004.04.011 